\title{
Peritoneal Flap Hernioplasty for Reconstruction of Large Ventral Hernias: Long-Term Outcome in 251 Patients
}

\author{
Michael Festersen Nielsen ${ }^{1}$ (i) $\cdot$ Andrew de Beaux ${ }^{1} \cdot$ Bruce Tulloh $^{1}$
}

Published online: 7 May 2019

(C) The Author(s) 2019

\begin{abstract}
Background Repair of large ventral hernias is challenging when primary fascial closure cannot be achieved. The peritoneal flap hernioplasty, a modification of the Rives-Stoppa retromuscular mesh repair, addresses this problem by using the hernial sac to bridge the fascial gap and isolate the mesh from both the intraperitoneal contents and the subcutaneous space. It is applicable to both midline and transverse hernias. We report the results from our institution using this repair based on a retrospective review of 251 cases.

Methods Patients undergoing peritoneal flap hernioplasty repair from January 1, 2010-December 31, 2014 were identified from the Lothian Surgical Audit system, a prospectively maintained computer database of all surgical procedures in the Edinburgh region of southeast Scotland. Patient demographics, clinical presentation, location of the hernia and surgical treatment were obtained from the hospital case-notes. Follow-up consisted of a clinical consultation 3 months postoperatively and a retrospective review of patient files completed December 2018. Patients presenting signs of complications were assessed during a clinical review.

Results Two hundred and fifty-one patients underwent incisional hernia repair, $68.1 \%$ in the midline and $31.9 \%$ arising through transverse incisions. Forty-three of these (17\%) were recurrences referred from other centers. Mean BMI was $32.1 \mathrm{~kg} / \mathrm{m}^{2}$ (range 20-59.4 kg/m ${ }^{2}$ ). Mean defect width was $9.2 \pm 4.2 \mathrm{~cm}$ (range 2.5-24.2 cm). Mean mesh size was $752 \mathrm{~cm}^{2}$ (range $150-1760 \mathrm{~cm}^{2}$ ). Some form of abdominoplasty was performed in $59 \%$ of cases. Mean postoperative stay was 6.3 days (range 1-33 days). Mean follow-up time was 75 months (range 44-104 months). Fifty-three patients $(21.1 \%)$ developed postoperative complications. Three (1.2\%) developed superficial skin necrosis and $27(10.8 \%)$ a superficial wound infection, but none developed deep mesh infection. Twelve (4.8\%) developed symptomatic seroma and $11(4.4 \%)$ a hematoma requiring surgical intervention. Seven (2.8\%) patients developed recurrence within the follow-up period.

Conclusion Peritoneal flap hernioplasty is an excellent and versatile method for reconstruction of large ventral hernias arising in both midline and transverse incisions. The technique is safe and associated with few complications and a very low recurrence rate.
\end{abstract}

Michael Festersen Nielsen

michael.nielsen@nhslothian.scot.nhs.uk

1 Department of Upper GI Surgery, Royal Infirmary of Edinburgh, 51 Little France Cres, Edinburgh EH16 4SA, Scotland, UK

\section{Introduction}

The repair of primary ventral and incisional hernias is common operations [1-3]. As the width of the defect increases, obtaining original fascia-to-fascia closure without excessive tension becomes more difficult. This is especially true for large ventral hernias, where primary 
closure can be difficult or impossible without the use of advanced surgical techniques [2, 4-7]. A number of techniques have been described to help manage this clinical situation such as bridging mesh (with poor outcomes), and component separation techniques [8-15].

An alternative method to component separation for reconstruction of large abdominal wall defects is the peritoneal flap technique. This method is based on the principles outlined for retromuscular mesh repair and is also known as the "Swinging Door" or "Mesh Sandwich" repair. It is widely used in the UK and Europe, and series have been published by Beck [16] and Malik et al. [17]. It utilizes redundant tissue from the hernial sac to bridge the facial gap, and it increases abdominal domain by expending the abdominal wall at the site of herniation without inducing weakness laterally. Because it reduces the need for extensive dissection, we consider this technique less traumatic and therefore likely associated with fewer complications. As opposed to component separation, which is applicable only to midline defects, the peritoneal flap technique may be used for reconstruction of both transverse and oblique as well as paramedian and midline incisional hernias.

The aim of this study is to present early and long-term results of the peritoneal flap technique in a single center based on a retrospective analysis of 251 patients undergoing ventral abdominal wall repair. Our review demonstrates that the peritoneal flap technique is associated with a low risk of early and long-term complications and a very low recurrence rate. We conclude that the technique is safe and versatile and should be used widely as an alternative to component separation.

\section{Materials and methods}

\section{Operative technique}

The peritoneal flap technique is here described for reconstruction of a midline incisional hernia (Fig. 1). The method can also be adapted for repair of transverse and oblique incisional hernias with careful attention to the anatomical layers of the abdominal wall at the site of the hernia.

The patient lies supine on the operating table. The previous midline scar is excised along with any redundant skin. Skin flaps are elevated no more than is needed to expose the sac, the defects margins and the entire length of the scar at the level of the deep fascia. The sac is then opened in the midline over the full length of the defect. No sac is excised at this point. Peritoneal adhesions within the sac and adjacent to the defect margins are divided, but

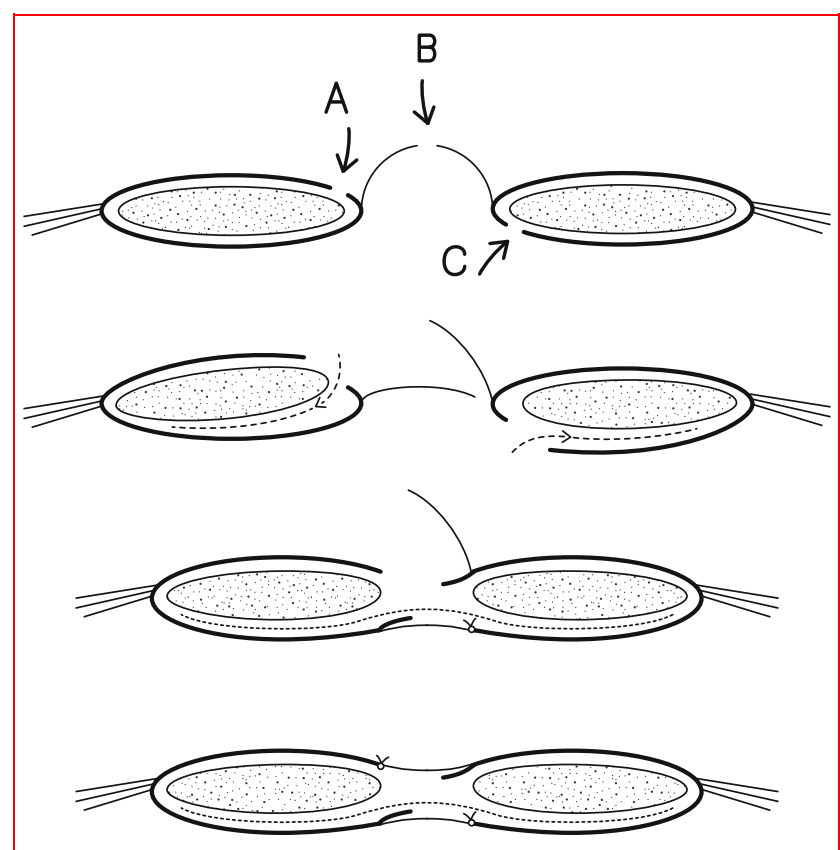

Fig. 1 Peritoneal flap repair. 1. The hernia sac is incised over the full length of the defect $(B)$ along with the anterior rectus sheath on one side $(A)$ and the posterior sheath on the other $(C)$. 2. The retromuscular spaces on each side are developed out to the linea semilunaris. 3. The flap of anterior sheath and sac from one side is transposed across the defect and sutured to the cut edge of the posterior sheath on the other side to close the abdominal cavity. A large piece of mesh can be laid in the retromuscualr space and fixed in position (dotted line). 4. The final step is closure of the remaining flap which isolates the mesh from the subcutaneous space

extensive intra-abdominal adhesiolysis is generally not required.

Mobilization of one peritoneal flap begins by incising the anterior rectus sheath on one side over the entire length of the wound in a line parallel to the margin of the defect, approximately $0.5 \mathrm{~cm}$ from the medial border of the rectus muscle. In cases with wide muscular diastasis, this incision may lie several centimeters away from the midline. Dissection continues around the medial border of the rectus muscle to enter the retromuscular space and in doing so the linea alba, with one half of the preserved sac still attached, "peels" off the muscle and advances medially for a centimeter or two. The retromuscular space can be developed as far laterally as the linea semilunaris to permit even more medial advancement. A flap comprising a little of the anterior sheath along with one half of the preserved hernia sac has thus been created and, because the anterior sheath has been divided and reflected around the medial border of the rectus muscle, it is now contiguous with the posterior sheath. This flap is destined to form the deep (posterior) layer of the repair.

On the opposite side of the wound, the musculofascial layer is elevated and a longitudinal incision is made 
through the parietal peritoneum and posterior rectus sheath, approximately $0.5 \mathrm{~cm}$ from the edge of the rectus muscle and over the full length of the wound but this time on the undersurface. The medial portion of the posterior sheath is reflected medially off the muscle and, with the other half of the sac still attached, now comes to lie anteriorly. This flap is destined to form the superficial layer of the repair. Once the retromuscular space is obtained, the dissection is continued laterally toward the linea semilunaris as in a standard Rives-Stoppa repair.

At this stage, the rectus muscles have been completely mobilized from the posterior sheaths on both sides. Care must be paid to the segmental neurovascular bundles entering the retromuscular space along the linea semilunaris.

At the superior and inferior reaches of the wound, the retromuscular spaces on each side must be joined across the midline. This is done by dividing the posterior sheaths as they insert into the undersurface of the linea alba, with care taken to ensure that the linea alba remains intact. Caudally, this dissection extends into the cavum Retzii behind the pubic bone (retropubic space), while cranially the dissection extends into the root of the falciform ligament and may continue beyond and deep to the xiphisternum.

Closure of the abdominal cavity is performed by suturing the posterior peritoneal flap (originally derived from the incision in the anterior sheath on one side) to the cut edge of the opposite posterior rectus sheath, underneath the muscle. If the peritoneal flap is very large, it may be trimmed as required to produce a neat, low-tension approximation. Once this is completed, the peritoneal cavity has been closed. The hernial defect is bridged by one peritoneal flap with minimal tension to create a common plane extending across the midline into the retro-rectus spaces on both sides. A suitable mesh is cut to size and laid into this space with at least $5 \mathrm{~cm}$ overlap above and below the closed peritoneal suture line and extending toward the linea semilunaris bilaterally. The mesh should be trimmed as required to lie flat with no folding or curling and no contact with the segmental nerves as they enter the muscle. It is fixed to the posterior sheath with interrupted sutures of a slowly absorbable monofilament material such as $2 / 0$ polydioxanone, four to six sutures on each side. Below the arcuate line, often no fixation is undertaken, although tissue glue may be used in this area to good effect. Alternatively, the mesh may be fixed with transfascial sutures to the anterior layers, or to Cooper's ligament deep in the pelvis.

The repair is finally completed by suturing the remaining peritoneal flap, originally derived from the posterior sheath but now reflected anteriorly, to the cut edge of the anterior sheath on the other side, overlying the rectus muscle. Again this flap may be trimmed to achieve a neat, low-tension closure. When this layer is completed, the mesh is covered on both its deep and superficial aspects by peritoneal sac in a "sandwich"-like fashion. The deep layer isolates the mesh from the peritoneal cavity, and the superficial flap protects the mesh from the subcutaneous plane. The repair also brings the rectus muscles closer together, although it does not necessarily restore them completely to the midline. With no damage incurred to the lateral musculofascial layers, the new slightly widened and mesh-reinforced linea alba allows the tension of the abdominal closure to be spread evenly across the whole abdominal wall.

Reconstruction of hernias arising through transverse or oblique incisions follows the same principles as outlined above, the main difference being that an appropriate tissue plane must be created lateral to the rectus sheath in which to place the mesh. Medially, the usual retro-rectus space is used. Laterally, the surgeon can choose either the plane between the external and internal oblique muscle layers as described by Stumpf et al. [18] or the extraperitoneal plane as described for a TAR repair $[8,9]$. The plane between internal oblique and transversus abdominis muscles should be avoided as this contains the segmental neurovascular structures. In practice, it is easier to develop the planes laterally and medially first, then finally join them together across the tissue junction at the lateral border of the rectus sheath. As for midline hernias, the sac is divided in the line of the incision and the retromuscular planes approached from anteriorly on one side and posteriorly on the other. The main principle of the dissection is to ensure that a wide common space is developed all around the hernia defect for mesh placement, and that enough sac is preserved to allow closure of these layers without undue tissue tension.

For both midline and non-midline hernia repairs, the skin incision may be closed in routine fashion once the anterior fascial layer has been closed over the mesh. Subcutaneous drains are recommended to reduce the risk of seroma, and drains to the plane of the mesh may be used at the surgeon's discretion.

\section{Medical records}

The present study is an uncontrolled case series from a single center. All patients undergoing a peritoneal flap hernioplasty repair in our institution from January 1, 2010, until December 31, 2014, were retrieved from the Lothian Surgical Audit (LSA) system, a prospectively maintained database of all surgical procedures in the Edinburgh region of southeast Scotland. Those undergoing elective repair for either midline or transverse anterior abdominal wall incisional hernias were identified from the database. Cases using other surgical techniques than the peritoneal flap hernioplasty were excluded, as were patients admitted for an emergency abdominal wall repair. The LSA database 
records primary referrals and notes from all patients assessed and treated for postoperative complications across all the hospital in the Lothian region. Complex abdominal wall repair is in Scotland considered a specialist procedure and all patients with potential complications following a repair performed in our institution are referred back to us for clinical assessment and treatment. Thus, the LSA database provides a reliable tool to accurately record readmissions and complications of the cases performed in our region. The review process for the study was conducted based on the clinical notes from the 3-month follow-up visit and subsequent LSA recordings from all 251 patients enrolled in the study.

Patient characteristics including age and sex of the patients, body mass index (BMI), operative details including defect size and mesh size, length of postoperative stay and postoperative progress at 3 months were recorded from each patient's case-notes (Table 1). Technical data with respect to the results from preoperative abdominal CT, length of hospital stay, mesh size and time of follow-up were likewise retrieved from patient files and recorded (Table 2). Postoperatively, patients' records were reviewed and assessed for short- and long-term complications (Table 3). Those with any symptoms or signs to suggest complications were either telephoned or clinically reviewed by a surgical consultant or a specialist registrar. Imaging (CT or ultrasound) was performed when needed to document the presence or absence of complications. Recurrence was defined as a hernia arising in the region of a previous abdominal wall repair, documented either during a clinical examination and/or by CT of the abdominal wall. Thus, it includes new hernias arising adjacent to a previous repair as well as true recurrences arising through the original fascial defect.

\section{Results}

Three hundred and fifteen open procedures for ventral and incisional hernia were performed by the two operating surgeons (BT and AdeB) from January 1, 2010, to

Table 1 Patient characteristics

\begin{tabular}{lll}
\hline Characteristics & Value $^{\mathrm{a}}$ & Range \\
\hline Number of patients included $(N)$ & 251 & \\
Gender distribution (male vs & 119 versus & $47.4 \%$ versus \\
$\quad$ female) & 132 & $52.6 \%$ \\
Age (years) & $59.3 \pm 12.3$ & $25-86$ \\
Height $(\mathrm{m})$ & $1.67 \pm 0.10$ & $1.47-1.93$ \\
Weight $(\mathrm{kg})$ & $88.8 \pm 20.8$ & $50-169$ \\
BMI $\left(\mathrm{kg} / \mathrm{m}^{2}\right)$ & $32.1 \pm 7.3$ & $20-59.4$ \\
\hline
\end{tabular}

${ }^{\mathrm{a}}$ Mean $\pm \mathrm{SD}$
Table 2 Technical data

\begin{tabular}{lll}
\hline Characteristics & Value $^{\mathrm{a}}$ & Range \\
\hline Previous hernia repair & $17.1 \%$ & \\
Incision (midline vs transverse) & $68.1 \%$ versus $31.9 \%$ & \\
Preoperative CT scan & $48.6 \%$ & \\
Abdominoplasty & $58.2 \%$ & \\
Mesh size $\left(\mathrm{cm}^{2}\right)$ & $752 \pm 312$ & $150-1760$ \\
Length of hospital stay (days) & $6.3 \pm 4.4$ & $1-33$ \\
Defect diameter on CT (cm) & $9.2 \pm 4.2$ & $2.5-24.2$ \\
Postoperative follow-up (months) & 75 & $44-104$ \\
\hline
\end{tabular}

${ }^{\mathrm{a}}$ Mean $\pm \mathrm{SD}$

Table 3 Postoperative complications

\begin{tabular}{lcc}
\hline Problem & Percent & Value $(\%)$ \\
\hline Skin necrosis & 3 & 1.2 \\
Superficial wound infection & 27 & 10.8 \\
Seroma requiring reoperation & 12 & 4.8 \\
Wound hematoma requiring reoperation & 11 & 4.4 \\
Chronic pain & 12 & 4.8 \\
Recurrence & 7 & 2.8 \\
\hline
\end{tabular}

December 31, 2014. In 288 of these (91\%), a peritoneal flap hernioplasty was performed. Two hundred and fiftyone patients fulfilled the inclusion criteria for elective repair and were recruited for the study (Table 1).

One hundred and thirty-two (52.6\%) were female and $119(47.4 \%)$ male. Patient characteristics are shown in Table 1. One hundred and seventy-one $(68.1 \%)$ were operated upon for a midline incisional hernia and 80 $(31.9 \%)$ for a transverse hernia. The size of the hernial defect was assessed from the operation report or from preoperative CT scans which were performed in $48.6 \%$ of cases. Mean maximum hernia width was $9.2 \mathrm{~cm}$ (range $2.5-24.2 \mathrm{~cm}$ ), and the mean size of mesh size used was $752 \mathrm{~cm}^{2}$ (range 150-1760 $\mathrm{cm}^{2}$ ). Abdominoplasty was performed in $146(58.2 \%)$ of cases (Table 2). Mean followup time was 75 months (range 44-104 months).

Early complications were recorded in $53(21.1 \%)$ case at the 3-month follow-up appointment, comprising 27 cases of superficial wound infection (10.6\%), 12 seromas (4.8\%) and $11(4.4 \%)$ hematomas requiring surgical intervention (Table 3). There were no cases of deep mesh infection. Superficial wound infections were treated with antibiotics, local drainage and/or surgical debridement and followed in the outpatient clinic. All cases had healed within 6 months of surgery. Seromas were treated with aspiration in the 
outpatient clinic, some requiring several aspirations until they resolved. Chronic pain defined as pain persisting for at least 3 months after surgery was recorded in $12(4.8 \%)$ of the patients. Skin necrosis was seen in three patients $(1.2 \%)$ and in one of these was extensive enough to involve the underlying fascia and peritoneal flaps, leading to mesh exposure. The wound was debrided and vacuum dressings applied until the patient was able to undergo revisional surgery.

Of the 251 patients included in the study, 40 patients had died at the time of follow-up. Thirty-seven of these from causes unrelated to the abdominal wall reconstruction. Two patients died prior to discharge, one from a stroke and one from myocardial infarction. One patient died from sepsis of unknown cause.

Seven patients $(2.8 \%)$ were identified as having a hernia recurrence. All seven have undergone surgery for recurrence by the time of closure of the study. The median BMI of this group of patients was $35.4 \mathrm{~kg} / \mathrm{m}^{2}$.

\section{Discussion}

Repair of ventral and incisional hernias by reinforcing primary fascial closure with a mesh in the retromuscular plane is commonly regarded as the current standard of care and is often referred to as the Rives-Stoppa-Wantz procedure [19-21]. Although widely applicable, this technique is not always appropriate for large hernias where primary fascial closure cannot be achieved owing to excessive tension and/or loss of abdominal domain. In such cases, a number of lateral tissue-releasing procedures have been described, which can be broadly grouped together as components separation techniques. In parts of Europe and the UK, the peritoneal flap hernioplasty is a popular alternative to component separation $[16,17]$. The peritoneal flap technique has evolved as a modification of the da Silva triple-layer repair, which used reflected sections of the anterior and posterior rectus sheaths as well as peritoneal sac to traverse the fascial gap reconstructing the midline in a low-tension manner while preserving the integrity of the lateral layers [22]. Abdominal domain is increased at the site of the herniation with native fascia and sac reinforced with mesh such that tension is distributed evenly across the whole abdominal wall. This is in contrast to the components separation techniques where tension is maximal laterally. The peritoneal flap hernioplasty adds a mesh to the da Silva repair, placing it in the retromuscular plane as in the Rives-Stoppa-Wantz repair. The peritoneal flap technique may therefore best be described as a hybrid of these two surgical procedures.

Anterior component separation, involving the release of the external oblique aponeurosis as first described by
Ramirez and colleagues in 1990 [23], remains popular as an adjunctive technique for repair of large ventral hernias. Various modifications have been described including endoscopic release of the external oblique muscle and more recently posterior component separation with transversus abdominis release (TAR) [3, 8, 11, 24]. The aim of these techniques is to facilitate medial approximation of the rectus muscles to achieve midline closure but each requires extensive dissection into the lateral planes of the abdominal wall and the sacrifice of one entire musculofascial layer. Seroma and superficial wound infections are common complications when large skin flaps are elevated, and recurrence rates in the region of $10-22 \%$ are reported [25-28]. While component separation does achieve tension-free midline closure, this is achieved at the expense of lateral abdominal wall integrity. To compensate for the lateral weakness induced by the release of the oblique or the transverse aponeurosis, a large mesh extending into the flanks may be used. This has the potential to affect the function of the abdominal wall from fibrosis induced by the mesh or from the extensive dissection itself. The peritoneal flap repair for midline hernias obviates these problems by avoiding dissection into the lateral abdominal wall altogether, using redundant tissue from the hernial sac to bridge the fascial gap and increase abdominal domain by expanding the abdominal wall at the site of herniation without inducing weakness laterally.

In contrast to component separation techniques, the peritoneal flap repair can be used to repair oblique and transverse abdominal wall defects in addition to those in the midline. In the present study, approximately $1 / 3$ of cases were for transverse incisional hernias, most often following open cholecystectomy or colonic resection. Also in the series were a number of L-shaped upper abdominal incisions used for liver transplantation and rooftop incisions used for Whipple's procedure and liver resection. The peritoneal flap repair in these patients involves developing a plane behind the rectus muscles medially, and between internal and external oblique muscles laterally then joining the two by dividing the linea semilunaris. The anatomy of this approach has been well described by Stumpf et al. [18]. Because the external oblique originates from the superficial part of the lower ribs above the costal margin, the dissection in this area may be continued up over the ribcage allowing the mesh to cover the primary defect with a large overlap. This is particularly useful in patients with defects located close to the costal margin where limited tissue is available for primary closure.

During the study period, the authors had not started using Botulinum-A therapy or preoperative pneumoperitoneum as adjuncts to preoperative workup in midline hernias to improve the chance of restoration of the midline. Further work is ongoing to see if such preoperative therapy 
can reduce the need for the peritoneal flap repair over conventional sublay repair.

Obesity has been found to be a risk factor for occurrence and recurrence of abdominal wall herniation, and it is known to increase the risk of infections and hematoma [29]. Owei and colleagues demonstrated that the risk of complications following anterior abdominal hernia repair is increased in patients with BMI above $30 \mathrm{~kg} / \mathrm{m}^{2}$, and doubled when BMI exceeds $40 \mathrm{~kg} / \mathrm{m}^{2}$ [30]. In the present study, median BMI was $30.1 \mathrm{~kg} / \mathrm{m}^{2}$, so half of the study subjects were obese at the time of operation (BMI $>30$ $\mathrm{kg} / \mathrm{m}^{2}$ ). Despite this, the rate of short-term complications in our patients was equivalent to what has been reported elsewhere in studies of patients undergoing abdominal wall repair. It is of interest to note that the mean BMI of the patients who developed recurrence $\left(35.4 \mathrm{~kg} / \mathrm{m}^{2}\right)$ was slightly higher than that observed for the whole group $\left(30.1 \mathrm{~kg} / \mathrm{m}^{2}\right)$. While this observation may imply that obesity per se is an independent risk factor for recurrence that association is likely to be weak, since only seven of the 251 repairs were treated for recurrence. Moreover, these recurrences were not clustered among the morbidly obese individuals, but occurred throughout the range of BMI (range $30.7-48.6 \mathrm{~kg} / \mathrm{m}^{2}$ ). Thus, we conclude that the peritoneal flap technique is a procedure that can be safely performed even in patients with morbid obesity, including combining abdominoplasty with the abdominal wall repair. Nevertheless, pre-habilitation with attention to weight reduction in such patients remains to be important [30].

The segmental nerves are put at risk when the dissection has been carried out into the lateral aspects of the abdominal wall. Often a concomitant bulging of the abdominal wall can be observed suggesting that these nerves may have been injured during the procedure. The segmental nerves and vessels perforate the rectus sheath close to the linea semilunaris where damage may occur during the retromuscular dissection, but nerve injuries may also arise from entrapment in suture material or scar tissue or by irritation from the mesh. We advocate careful dissection leaving nerves and muscle intact is likely to be important to reduce the incidence of pain following this repair.

The importance of restoration of the midline rectus musculature to regain physiological function of the abdominal wall remains open to debate. A main objective for both anterior and posterior component separation is to achieve midline fascial closure, but the peritoneal flap hernioplasty does not attempt this. Even so, it does approximate them to a considerable degree (Fig. 2). It is our contention that abdominal wall function relies more on an intact circle or ring of abdominal muscle/fascia, and that it does not matter if the gap between the muscles is several centimeters wide as long as it is firm and strong, providing a firm ridge of fibrous tissue against which the recti and lateral obliques can pull. There is no doubt that the triplelayer neo-linea alba produced in a peritoneal flap repair provides this. Indeed, as the results from the 3-month clinical review appointment reveal, there is a high degree of patient satisfaction after the peritoneal flap repair, with very few complaints of bulge or other complaints related to the appearance or weakening of the abdominal wall. Indeed, those with significant 'divarication' preoperatively do not manifest the typical midline bulge when initiating a sit-up maneuver.

In conclusion, the present paper reports the results from a series of ventral and incisional hernia repairs using the
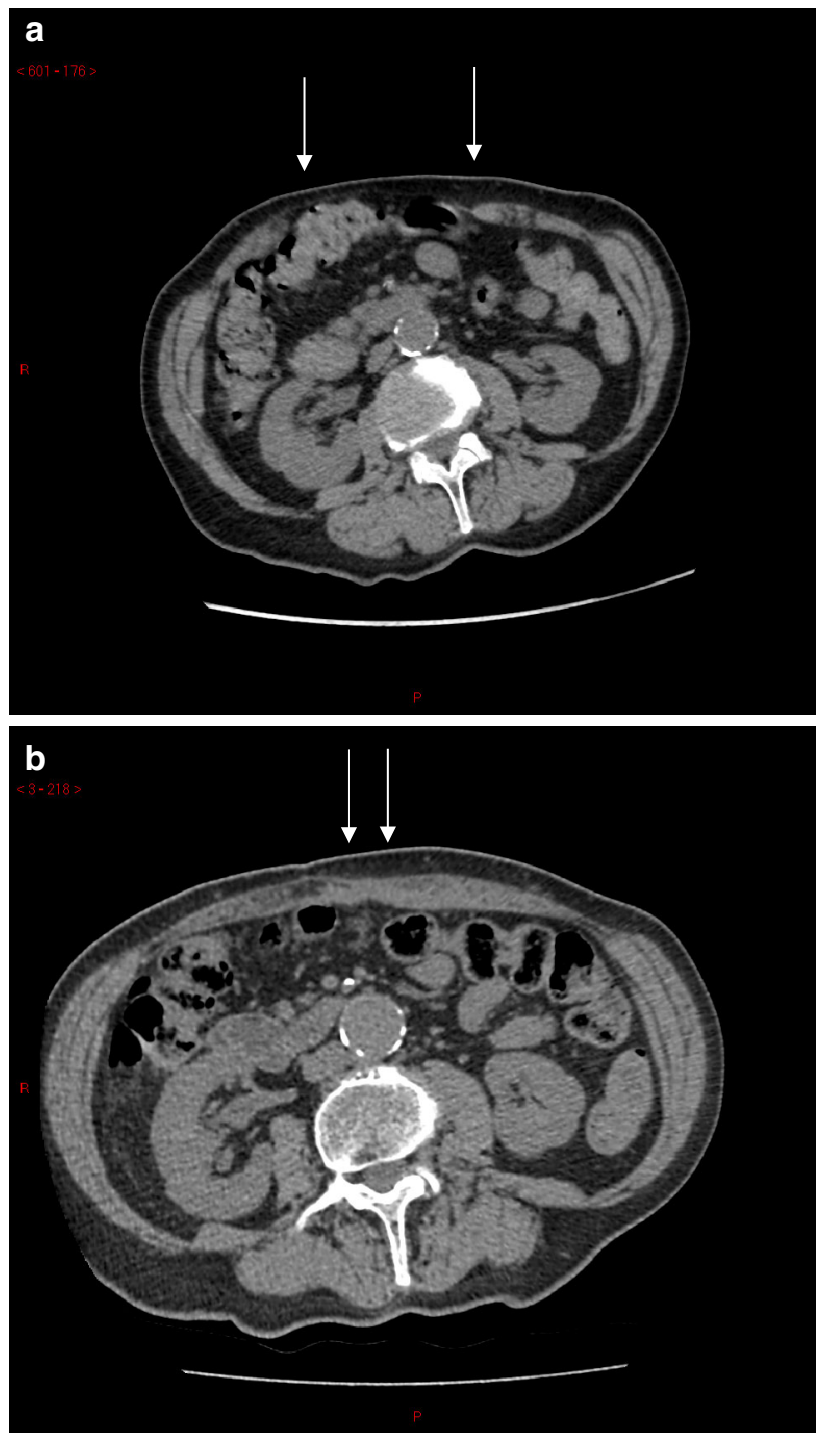

Fig. 2 Preoperative and postoperative CT scans. The defect of $11 \mathrm{~cm}$ width (a) is reduced to no more than $3 \mathrm{~cm}$, with a thick and strong neo-linea alba, on a CT scan taken 7 months postoperatively for unrelated reasons $(\mathbf{b})$ 
peritoneal flap hernioplasty. Our retrospective analysis reveals excellent short- and long-term outcomes in a series of 251 peritoneal flap hernioplasties at one center after a median follow-up of 75 months. No procedure-related mortality was observed. Seven patients were diagnosed and treated for recurrence resulting in a long-term recurrence rate of $2.8 \%$. These results demonstrate that the peritoneal flap hernioplasty is a safe and versatile procedure associated with low complication rates and a very low recurrence rate. Based on these results we recommend that the peritoneal flap hernioplasty be considered as an alternative to component separation in large hernias where primary fascial closure is difficult to achieve.

Open Access This article is distributed under the terms of the Creative Commons Attribution 4.0 International License (http://crea tivecommons.org/licenses/by/4.0/), which permits unrestricted use, distribution, and reproduction in any medium, provided you give appropriate credit to the original author(s) and the source, provide a link to the Creative Commons license, and indicate if changes were made.

\section{References}

1. Bucknall T, Cox P, Ellis H (1982) Burst abdomen and incisional henia: a prospective study of 1129 major laparotomies. Br Med J 1982:931-933

2. Mudge M, Hughes L (1985) Incisional hernia: a 10-year prospective study of incidence and attitudes. Br J Surg 72:70-71

3. Pauli E, Rosen M (2013) Open ventral henia repair with component separation. Surg Clin N Am 93:1111-1133

4. Cassar K, Munro A (2002) Surgical treatment of incisional hernia. Br J Surg 89:534-545

5. Paul A, Korenkov M, Peters S (1998) Unacceptable results of the Mayo procedure for repair of abdominal incisional hernias. Eur J Surg 164:361-367

6. Burger J, Luijendijk R, Hop W (2004) Long-term follow-up of a randomized controlled trial of suture versus mesh repair of incisional hernia. Ann Surg 240:578-583

7. Hesselink V, Luijendijk R, de Wilt J et al (1993) An evaluation of risk factors in incisional hernia recurrence. Surg Gynecol Obstet 176:228-234

8. Pauli E, Wang J, Petro CC et al (2015) Posterior component separation with transversus abdominis release successfully addresses recurrent ventral hernias following anterior component separation. Hernia 19:285-291

9. Jones C, Winder J, Potochy J et al (2016) Posterior component separation with transversus abdominis release: technique, utility and outcomes in complex abdominal wall reconstruction. Plast Reconstr Surg 137:636-646

10. Oprea V, Radu V, Moga D (2016) Transversus abdominis release (TAR) for large incisional hernia repair. Chirurgia 11:535-540

11. Novitsky YW, Elliott H, Orenstein S et al (2012) Transversus abdominis muscle release: a novel approach to posterior component separation during complex abdominal wall reconstruction. Am J Surg 204:709-716
12. Heller L, McNichols C, Ramirez O (2012) Component separation. Semin Plast Surg 26:25-28

13. Alam N, Narang S, Pathak S et al (2016) Methods of abdominal wall expansion for repair of incisional hernia: a systemic review. Hernia 20:191-199

14. Hauters P, Desmet J, Gherardi D et al (2017) Assessment of predictive factors for recurrence in laparoscopic ventral henia repair using bridging technique. Surg Endosc 31:3656-3663

15. Holihan J, Askenasy E, Greenberg J et al (2016) Component separation vs bridged repair for large ventral henias: a multiinstitutional risk-adjusted comparison systematic review and meta analysis. Surg Infect 17:17-26

16. Beck M (2011) Grandes eventrations autoplastic et prothese retromusculaire. In: Avci C, Foutanier G, Avtan L (eds). VideoAtlas Chirurgie herniaire, 1st edn. Springer, Berlin. ISBN 978-8178-0144-5

17. Malik A, Macdonald A, de Beaux A et al (2014) The peritoneal flap hernioplasty for repair of large ventral and incisioan hernias. Hernia 18:39-45

18. Stumpf M, Conze J, Prescher A et al (2009) The lateral incisional hernia: anatomical considerations for a standardized retromuscular sublay repair. Hernia 13:293-297

19. Stoppa R, Louis D, Verhaeghe P et al (1987) Current surgical treatment of post-operative eventrations. Int Surg 72:42-44

20. Heartsill L, Richards M, Arfai N et al (2005) Open Rives-Stoppa ventral hernia repair made simple and succesful but not for everyone. Hernia 9:162-166

21. Bauer J, Harris M, Gorfine S et al (2002) Rives-Stoppa procedure for repair of large incisional hernias: experience with 57 patients. Hernia 6:120-123

22. Da Silva A (1979) Surgical correction of longitudinal median and paramedian incisional hernia. Surg Gynecol Obstet 148:579-583

23. Ramirez O, Ruas E, Dellon L (1990) "Component separation" method for closure of abdominal-wall defects: an anatomic and clinical study. Plast Reconstr Surg 86:519-526

24. Nockolds C, Hodde J, Rooney P (2014) Abdominal wall reconstruction with components separation and mesh reinforcement in complex henia repair. BMC Surg 14:1-7

25. Jr Lowe, Lowe J, Baty J (2003) Risks associated with "component separation" for closure of complex abdominal wall defects. Plast Reconstr Surg 111:1276-1283

26. Hultman C, Tong W, Kittinger B (2011) Management of recurrent hernia after component separation: 10-year experience with abdominal wall reconstruction at an academic medical center. Ann Plast Surg 66:504-507

27. Ko J, Wang E, Salway D (2009) Abdominal wall reconstruction: lessons learned from 200 "component separation" procedures. Arch Surg 144:1047-1055

28. Deerenberg E, Timmermans L, Hogerseil D et al (2015) A systematic review of the surgical treatment of large incisional hernia. Hernia 19:89-101

29. Sauerland S, Korenkov M, Kleinen T et al (2004) Obesity is a risk factor for recurrence after incisional hernia repair. Hernia 8:42-46

30. Owei L, Swendiman RA, Kelz RR et al (2017) Impact of body mass index on open ventral hernia repair: a retrospective review. Surgery $162: 1320-1329$

Publisher's Note Springer Nature remains neutral with regard to jurisdictional claims in published maps and institutional affiliations. 